\title{
Composición y estructura de la vegetación de los bosques esclerófilos de la Selva Central del Perú
}

\author{
Composition and vegetation structure of the sclerophyllous forests \\ of the Selva Central of Peru
}

\author{
Mayra Briceño Huayta ${ }^{1, *}$
}

\begin{abstract}
Resumen
Los bosques esclerófilos son bosques achaparrados conformados principalmente por arbustos, que crecen sumamente entrelazados, lo que los convierte en una vegetación densa. Se evaluaron 12 parcelas ( 0.1 ha cada una) en 6 sitios de muestreo ( 2 parcelas por cada sitio), ubicadas en el Parque Nacional Yanachaga Chemillén (5 sitios); y ACM Bosque Sho'llet (1 sitio). La vegetación leñosa estuvo conformada por 35 familias, 61 géneros y 162 especies de plantas vasculares. Las familias con mayor riqueza de especies fueron Melastomataceae (21) y Asteraceae (19) y las familias con mayor abundancia fueron Clusiaceae (3128) y Primulaceae (1095). La distribución diamétrica de la vegetación leñosa se concentró entre 1-4.99 cm para el $92.12 \%$ de los individuos, de los cuales el 55.28\% tuvieron entre 1-1.99 cm de diámetro. Las cinco familias con mayor importancia ecológica basada en la abundancia, área basal y frecuencia relativa fueron: Clusiaceae (58.730), Chloranthaceae (25.095), Primulaceae (24.799), Cunoniaceae (23.224) y Pentaphylacaceae (20.629). El 73.13\% de la vegetación leñosa tuvo menos de $5 \mathrm{~m}$ de alto, de los cuales el 59.65\% estuvo en un rango de $2-3 \mathrm{~m}$ de alto. El análisis de ordenación basada en el índice de Morisita Horn demostró una baja similitud entre los seis sitios evaluados (Stress 0.1763).
\end{abstract}

Palabras clave: bosque esclerófilo, bosque achaparrado, bosque enano, selva central, Yanachaga, Oxapampa.

\footnotetext{
${ }^{1}$ Jardín Botánico de Missouri, Prolog. Bolognesi Mz. E, Lote 6, Oxapampa, Pasco, Perú.

* Autor de Correspondencia: ebhmayra@gmail.com
} 


\begin{abstract}
Sclerophyllous forests are scrub forests consisting mainly of shrubs, which grow extremely intertwined and makes them dense vegetation. There were installed 12 plots (0.1 ha each) distributed in 6 sites ( 2 plots per site), located in the Yanachaga Chemillen National Park (5 sites); and ACM Bosque Sho'llet (1 site). The flora composition of the woody vegetation is made up of 35 families, 61 genera and 162 species of vascular plants. The families with the highest species richness were Melastomataceae (21), and Asteraceae (19) and the families with the highest abundance were Clusiaceae (3128) and Primulaceae (1095). The diameter distribution of the woody vegetation was concentrated on $1-4.99 \mathrm{~cm}$ for $92.12 \%$ of the individuals, which $55.28 \%$ of them had $1-1.99 \mathrm{~cm}$ in diameter. The five families with greater ecological importance based on abundance, basal area and relative frequency were Clusiaceae $(58,730)$, Chloranthaceae $(25,095)$, Primulaceae $(24,799)$, Cunoniaceae $(23,224)$ and Pentaphylacaceae $(20,629)$. The $73.13 \%$ of the woody vegetation had less than $5 \mathrm{~m}$ high, of which $59.65 \%$ had $2-3 \mathrm{~m}$ high. The ordination analysis based on the Morisita Horn index showed a low similarity among the six sites (Stress 0.1763).
\end{abstract}

Key words: sclerophyll forest, elfin forest, dwarf forest, selva central, Yanachaga, Oxapampa.

\section{Introducción}

Los bosques esclerófilos se caracterizan por poseer una vegetación achaparrada, con abundantes arbustos delgados de $2 \mathrm{~m}$ de alto en promedio (Vásquez et al. 2005), y pocos árboles de hasta $5 \mathrm{~m}$, los cuales crecen entrelazados y/o sumamente ramificados, convirtiéndola en una vegetación densa y difícil de ingresar. El término esclerófilo deriva de los vocablos griegos $E s$ clero (duro) y Filo (hoja), y ha sido añadido por la abundante presencia de especies con hojas coriáceas debido al gran desarrollo que alcanza el esclerénquima, tejido constituido por células totalmente engrosadas y lignificadas (Font Quer 1953).

Este tipo de vegetación usualmente se desarrolla sobre un suelo de turbera y abundante musgo, principalmente Sphagnum; que le dan una apariencia de "almohadillas". Igualmente, los arbustos y árboles están cubiertos por este musgo; además de líquenes, hepáticas y plantas epífitas vasculares. Estos bosques se desarrollan en las cumbres de las montañas, después de los bosques nublados, y presentan abundantes precipitaciones y nubosidad durante gran parte del año.
En la Selva Central del Perú este tipo de vegetación se puede encontrar principalmente en el Parque Nacional Yanachaga Chemillén (PNYCh), y en el Área de Conservación Municipal Bosque Sho'llet. El PNYCh a lo largo de su gradiente altitudinal (370-3800 msnm) presenta una amplia variedad de formaciones vegetales, entre ellas se encuentran los bosques esclerófilos, distribuidos a modo de islas (SERNANP 2015). Asimismo, el Área de Conservación Municipal Bosque Sho'llet, se ubica en la zona sur de la zona de amortiguamiento del PNYCh, donde se pueden encontrar principalmente bosques esclerófilos y bosques montano alto.

Estos bosques esclerófilos proveen de servicios ecosistémicos a las poblaciones locales, cumplen un rol fundamental en el ciclo hidrológico del agua debido a su ubicación en las cabeceras de cuenca, y contienen un alto potencial de especies todavía no descritas por la ciencia; pues se han publicado cerca de veinte de especies nuevas de plantas arbóreas en las últimas dos décadas provenientes del PNYCh (Fernandez-Hilario y Bravo en prep.), muchas de ellas endémicas. Además, estos bosques 
constituyen el hábitat principal del Oso de anteojos (Tremarctos ornatus), categorizado en estado vulnerable a nivel nacional e internacional (DS No 004-2014-MINAGRI y UICN respectivamente). Debido a ello, el presente estudio tiene por objetivo contribuir a resolver las siguientes preguntas: ¿cuál es la composición de la flora de los bosques esclerófilos ubicados en el PNYCh, Bosque Sho'llet y zonas aledañas?, ¿cuál es la estructura de la vegetación de los bosques esclerófilos que se desarrollan en estas áreas protegidas y en zonas aledañas? y ¿cuál es la similitud entre los sitios evaluados?

\section{Materiales y Métodos}

\section{Área de estudio}

La presente investigación se ha llevado a cabo en dos áreas protegidas: (1) Parque Nacional Yanachaga Chemillén (PNYCh), y (2) Área de Conservación Municipal Bosque Sho'llet (en adelante ACM Bosque Sho'llet); y en zonas aledañas a las mismas (ver Figura 1).

El PNYCh se localiza en la selva central del Perú, comprende los distritos de Oxapampa, Huancabamba, Pozuzo y Villa Rica, provincia de Oxapampa, región Pasco. Se extiende sobre la aislada Cordillera del Yanachaga en la vertiente oriental; su rango altitudinal varía desde los 370 msnm en los bosques amazónicos, hasta los $3800 \mathrm{msnm}$ en la puna (SERNANP 2015). El ACM Bosque Sho'llet, se ubica en el límite norte de los distritos de Oxapampa y Villa Rica. Se extiende sobre los $2200 \mathrm{msnm}$ (ProNaturaleza s/f).

Se ubicaron seis sitios de muestreo donde se distribuye esta comunidad vegetal, de los cuales cuatro sitios se encuentran dentro del PNYCh: Oso Playa (S1), Abra Esperanza (S2), San Daniel (S4) y Abra Yanachaga (S6); un sitio fuera del PNYCh, Milpo (S3); y un sitio ubicado en el ACM Bosque Sho'llet (S5) (ver Figura 1). En este último sitio, una de las parcelas se ubicó fuera del ACM (ver Cuadro 1).

\section{Método de muestreo}

Se utilizaron las Parcelas Modificadas de Whittaker, que consisten en parcelas de 0,1 ha (50 x $20 \mathrm{~m})$ (Campbel et al. 2002). Cada parcela fue dividida en 5 sub unidades de $10 \times 20 \mathrm{~m}$. Se instalaron 2 parcelas de 0,1 ha en cada sitio, haciendo un total de 12 parcelas.

\begin{tabular}{|c|c|c|c|c|c|c|}
\hline \multirow{2}{*}{ Sitio } & \multirow{2}{*}{ Ubicación } & \multirow{2}{*}{$\begin{array}{l}\text { Nombre de } \\
\text { sitio }\end{array}$} & \multirow{2}{*}{$\mathrm{N}^{0}$ parcela } & \multicolumn{2}{|c|}{ Coordenadas } & \multirow{2}{*}{$\begin{array}{l}\text { Altitud } \\
(\mathbf{m s n m})\end{array}$} \\
\hline & & & & & $8 \mathrm{~L})$ & \\
\hline \multirow{2}{*}{$\mathrm{S} 1$} & \multirow{2}{*}{ PNYCh } & \multirow{2}{*}{ Oso Playa } & Parcela 1 & 434210 & 8858909 & 2504 \\
\hline & & & Parcela 2 & 434307 & 8859062 & 2561 \\
\hline \multirow{2}{*}{$\mathrm{S} 2$} & \multirow{2}{*}{ PNYCh } & \multirow{2}{*}{ Abra Esperanza } & Parcela 3 & 461770 & 8834707 & 2968 \\
\hline & & & Parcela 4 & 461231 & 8836120 & 2916 \\
\hline \multirow{2}{*}{$\mathrm{S} 3$} & \multirow{2}{*}{$\begin{array}{l}\text { Fuera del } \\
\text { PNYCh }\end{array}$} & \multirow{2}{*}{ Milpo } & Parcela 5 & 431656 & 8852312 & 2974 \\
\hline & & & Parcela 6 & 431845 & 8852092 & 2900 \\
\hline \multirow{2}{*}{ S4 } & \multirow{2}{*}{ PNYCh } & \multirow{2}{*}{ San Daniel } & Parcela 7 & 449891 & 8846397 & 2367 \\
\hline & & & Parcela 8 & 449831 & 8846197 & 2362 \\
\hline \multirow{2}{*}{ S5 } & \multirow{2}{*}{$\begin{array}{l}\text { ACM y fuera } \\
\text { del ACM }\end{array}$} & \multirow{2}{*}{$\begin{array}{l}\text { ACM Bosque } \\
\text { Sho'llet }\end{array}$} & Parcela 9 & 465199 & 8819784 & 2419 \\
\hline & & & Parcela 10 & 468668 & 8819846 & 2433 \\
\hline \multirow{2}{*}{ S6 } & \multirow{2}{*}{ PNYCh } & \multirow{2}{*}{$\begin{array}{l}\text { Abra Yana- } \\
\text { chaga }\end{array}$} & Parcela 11 & 449479 & 8852430 & 2944 \\
\hline & & & Parcela 12 & 449524 & 8852343 & 2992 \\
\hline
\end{tabular}

Cuadro 1. Localización de los sitios y las parcelas evaluadas. 


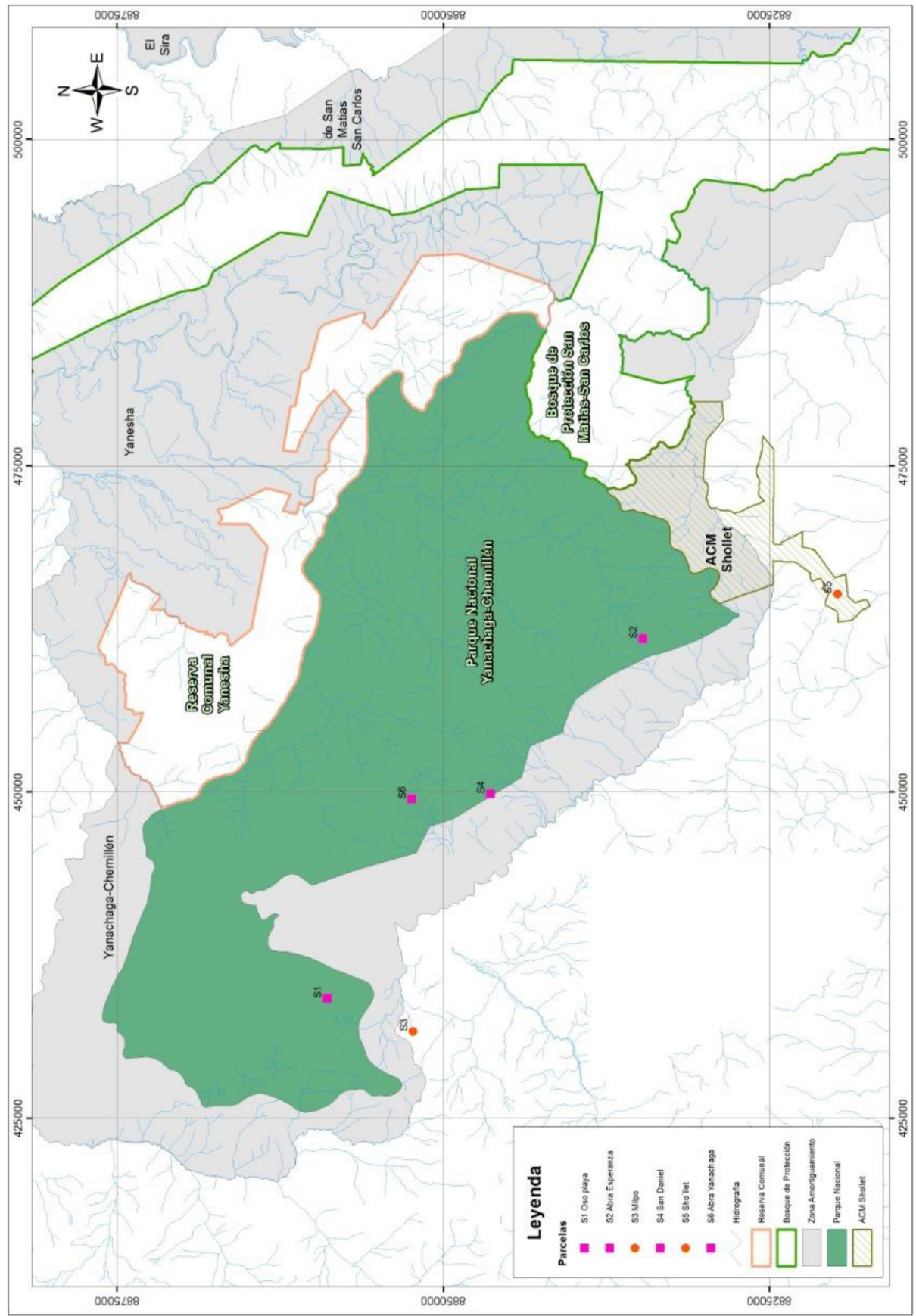

Figura 1. Mapa de ubicación de los sitios evaluados. 
En el muestreo se incluyó a todos los individuos de tallo leñoso (árboles, arbustos y lianas) con un DAP $\geq 1 \mathrm{~cm}$ a $1.30 \mathrm{~m}$ de alto, y se tomaron datos de altura de los individuos. Se optó por medir individuos leñosos a partir de $1 \mathrm{~cm}$ de DAP debido a su abundancia, y su implicancia en la estructura de estas comunidades vegetales. Para criterios específicos de medición se tomó como referencia el Manual de campo para la Establecimiento y Remedición de parcelas de RAINFOR de Philip \& Baker (2002). Se colectaron especímenes por duplicados, los cuales fueron depositados en los Herbarios HOXA, USM, MO y MOL.

Para la identificación de los especímenes, el material botánico colectado fue confrontado con las colecciones del Herbario HOXA del Jardín Botánico de Missouri en la ciudad de Oxapampa y en el Herbario USM del Museo de Historia Natural de la Universidad Nacional Mayor de San Marcos en Lima. Asimismo, se utilizó bibliografía física y digital especializada, como Trópicos del Missouri Botanical Garden (http://www.tropicos.org/NameSearch.aspx) y Neotropical Herbarium del Field Museum of Chicago (https:// plantidtools.fieldmuseum.org/en/rrc/5305). Para la correcta determinación taxonómica de las especies se siguieron los criterios de Angiosperm Phylogeny Group IV (APG IV 2016).

\section{Análisis de Datos}

Curva de acumulación de especies, para analizar si la riqueza de especies reportada ha sido suficiente para registrar una muestra representativa de las especies que se desarrollan en estos bosques esclerófilos, se ha elaborado una curva de acumulación de especies utilizando estimadores no paramétricos. Se utilizaron dos estimadores no paramétricos: Chao 2 (Colwell y Coddington 1994) y Jacknife de primer orden (Palmer 1990) los cuales toman en cuenta la distribución de las especies a través del muestreo (Colwell y Coddington 1994; Magurran 2004). Para este análisis se utilizó el programa EstimateS version 9.1.0 (Colwell 2013).

Composición florística, se elaboró una lista de todas las familias, géneros y especies leñosas registradas en cada uno de los seis sitios de muestreo (ver Anexo 1).
Riqueza y abundancia, se calculó la riqueza específica de especies (S) mediante el número de especies registradas por cada parcela (unidad de muestreo), por sitio de muestreo, y por cada taxa a nivel de familia, y la abundancia de las especies $(\mathrm{N})$ según el número de individuos registrados para cada uno de los elementos ya mencionados.

Estructura de la vegetación, para analizar la estructura de esta comunidad vegetal se ha determinado el Índice de Valor de Importancia (IVI) mediante la suma de las variables: frecuencia relativa, densidad relativa y área basal relativa, resultando el valor máximo de 300, mientras más próximo a este valor se encuentre un taxón, mayor será su importancia ecológica. Adicionalmente, se analizó la estructura horizontal a través de clases diamétricas. Se establecieron las siguientes clases: $1-1.99 \mathrm{~cm}$; 2-4.99 cm; 5-9.99 cm; 10-19.99 cm; 20-29.99 $\mathrm{cm} ; 30-40 \mathrm{~cm}$. La estructura vertical se representó mediante unos diagramas de cajas elaborado en Past v3.16 (Hammer et al. 2001); basado en los datos de alturas de los individuos registrados por cada sitio. Cada caja contiene una línea horizontal que representa el promedio.

Análisis de ordenación, para evaluar la similitud entre las parcelas y sitios de muestreo se utilizó el Escalamiento Multidimensional No Métrico (NMDS), debido a que no supone una relación lineal entre las variables, es iterativo y busca posicionar las variables de acuerdo a sus distancias originales (Hammer 1999-2018), permite utilizar una medida de similitud, y determina el nivel de Stress en la ordenación de los datos. En este caso se escogió utilizar el índice de Morisita Horn debido a que es cuantitativo y relaciona la abundancias específicas con las abundancias relativas y el total (Villareal et al. 2006). Para este análisis se usó el programa Past v3.16 (Hammer et al. 2001).

\section{Resultados}

\section{Curva de acumulación de especies}

Para el presente estudio se han evaluado 12 parcelas a lo largo de 6 sitios de muestreo (S1-S6). Para conocer si las especies registra- 

esclerófilos de la Selva Central del Perú

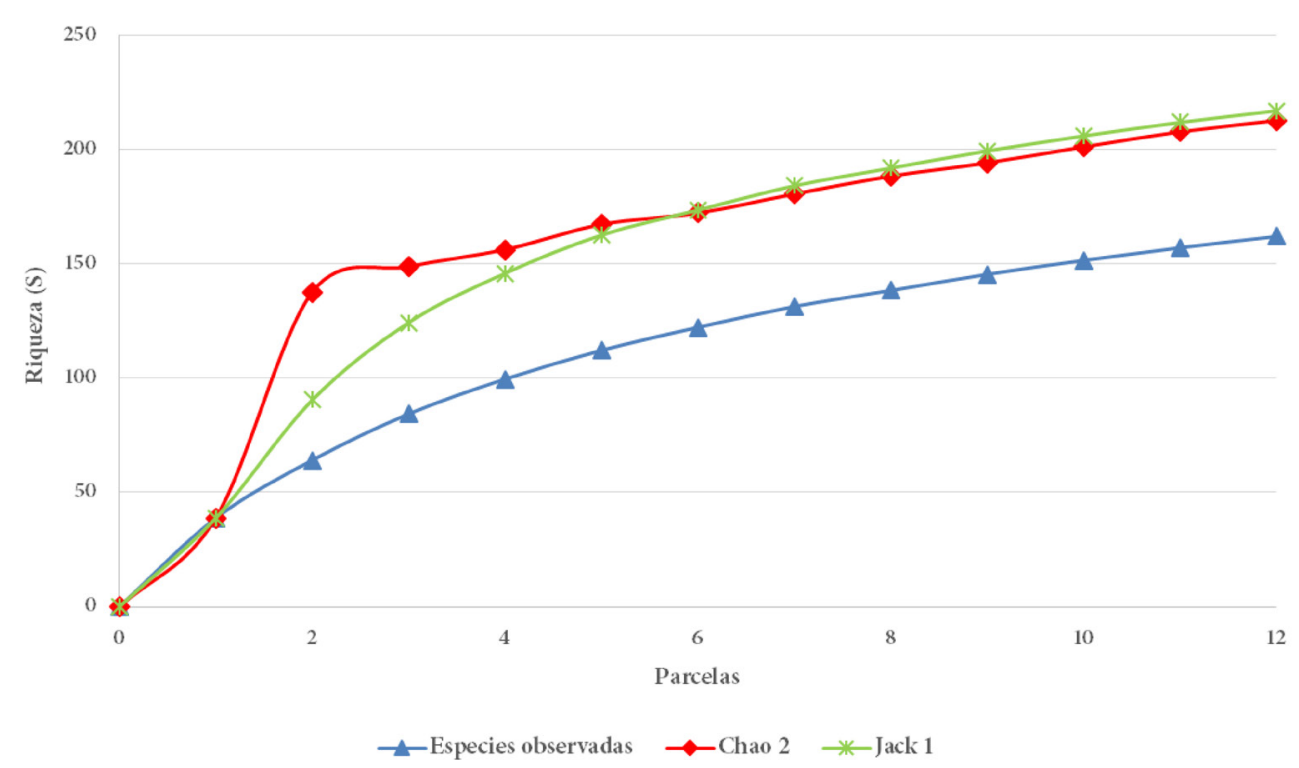

Figura 2. Curva de acumulación de especies en base a las especies observadas y esperadas.

das contienen una muestra representativa de la riqueza encontrada en estas comunidades vegetales se ha elaborado una curva de acumulación de especies en base a las especies observadas y dos estimadores no paramétricos Chao 2 y Jacknife 1 que brindan una aproximación de la riqueza esperada del área. Las especies registradas en los seis sitios de muestreo fueron 162 , entre árboles, arbustos y lianas, mientras que, las especies esperadas de acuerdo a Chao 2 fueron 212.7, y de acuerdo a Jacknife 1 fueron 217. Según estos estimadores, la riqueza de especies observada representó el 76.16\% y $74.65 \%$ del total de especies que componen los bosques esclerófilos, respectivamente.

\section{Riqueza y abundancia}

La composición de la flora leñosa de los bosques esclerófilos consiste de 35 familias, 61 géneros, 162 especies y morfoespecies (Cuadro 2), distribuidas en 9578 individuos registrados con un DAP $\geq 1 \mathrm{~cm}$. En este registro, se incluyeron 4 especies de helechos arbóreos, así como 3 especies de Arecaceae (monocotiledónea) como parte de la vegetación leñosa. En cuanto al hábito o forma de crecimiento, se han registrado lianas de los géneros Smilax y Mikania, el restante de las especies leñosas son arbóreas, arbustivas o subarbustivas.

La familia con mayor registro de especies fue Melastomataceae con 21 especies, seguida de Asteraceae (19), Ericaceae (18), Cunoniaceae (16), Aquifoliaceae (12), Clusiaceae (9), Primulaceae (7), Symplocaceae (6), Rubiaceae (5), y Pentaphylaceae (4). Estas 10 familias en conjunto representaron el $71.78 \%$ del total de las especies registradas.

En cuanto al número de individuos, este estuvo distribuido en las siguientes 10 familias: Clusiaceae (3128), Primulaceae (1095), Melastomataceae (1024), Chloranthaceae (764), Ericaceae (624), Asteraceae (570), Cunoniaceae (569), Pentaphylacaceae (336), Aquifoliaceae (270) y Symplocaceae (269), las cuales representaron el $90.28 \%$ de los individuos registrados (Cuadro 3).

Si analizamos la riqueza y abundancia por cada sitio de muestreo (ver Figura 3); y a su vez por cada parcela de muestreo (ver Figura 4); en el Abra Esperanza (S2) se registró la mayor riqueza de especies (69 especies para las dos 


\begin{tabular}{|c|c|c|c|}
\hline Grupo & Familias & Géneros & Especies \\
\hline Eudicotiledóneas & 31 & 56 & 154 \\
\hline Monocotiledóneas & 1 & 2 & 3 \\
\hline Gymnospermas & 1 & 1 & 1 \\
\hline Pteridofitas & 2 & 2 & 4 \\
\hline Total & $\mathbf{3 5}$ & $\mathbf{6 1}$ & $\mathbf{1 6 2}$ \\
\hline
\end{tabular}

Cuadro 2. Registro de las especies de vegetación leñosa en los seis sitios de muestreo de bosques esclerófilos.

parcelas) de los seis sitios de muestreo; en tan solo una de las parcelas se registraron 53 especies (parcela 03); y en la otra parcela 44 especies (parcela 04). Asimismo, en el ACM Bosque Sho'llet (S5) se registraron 59 especies (para las dos parcelas), ocupando el segundo lugar en número de especies de los seis sitios, en una de sus parcelas se registraron 51 especies (parcela 9); y en la otra 36 especies (parcela 10). En San Daniel (S4) se registró la menor riqueza de especies (38 especies en dos parcelas); en una de sus parcelas se encontró tan solo 19 especies (parcela 07), mínimo registro a nivel de las 12 parcelas; y en la otra 33 especies (parcela 08).

Es notoria la diferencia en la riqueza de especies de las parcelas en los dos últimos sitios mencionados: ACM Bosque Sho'llet (S5) y San Daniel (S4). En el ACM Bosque Sho'llet (S5) la diferencia en la riqueza entre las dos parcelas es de 15 especies, mientras que, en San Daniel (S4), la diferencia entre las parcelas es de 14 especies.

En cuanto a la abundancia (ver Figura 3 y Figura 4), en Oso Playa (S1) se registraron 3230 individuos, el mayor número de individuos de los seis sitios, mientras que, en Milpo (S3), fueron registrados 480 individuos (Figura 03). Cabe mencionar que a nivel de parcelas para el caso de Oso Playa (S1), en tan solo una de las parcelas se registraron 2347 individuos

Cuadro 3. Riqueza (S) y abundancia $(\mathrm{N})$ por familias registradas en los seis sitios de muestreo de bosques esclerófilos.

\begin{tabular}{|c|c|c|}
\hline Familia & Riqueza (S) & Individuos (N) \\
\hline Adoxaceae & 2 & 10 \\
\hline Alzateaceae & 1 & 37 \\
\hline Aquifoliaceae & 12 & 270 \\
\hline Araliaceae & 3 & 62 \\
\hline Arecaceae & 3 & 11 \\
\hline Asteraceae & 19 & 570 \\
\hline Berberidaceae & 1 & 3 \\
\hline Brunelliaceae & 2 & 22 \\
\hline Campanulaceae & 1 & 8 \\
\hline Caprifoliaceae & 1 & 2 \\
\hline Chloranthaceae & 3 & 764 \\
\hline Clethraceae & 1 & 58 \\
\hline Clusiaceae & 9 & 3128 \\
\hline Columelliaceae & 1 & 21 \\
\hline Culcitaceae & 1 & 1 \\
\hline Cunoniaceae & 16 & 569 \\
\hline Cyatheaceae & 3 & 68 \\
\hline Ericaceae & 18 & 624 \\
\hline Gentianaceae & 3 & 42 \\
\hline Hypericaceae & 1 & 2 \\
\hline Lauraceae & 4 & 99 \\
\hline Loranthaceae & 1 & 188 \\
\hline Melastomataceae & 21 & 1024 \\
\hline Myricaceae & 1 & 1 \\
\hline Myrtaceae & 2 & 151 \\
\hline Pentaphylacaceae & 4 & 336 \\
\hline Podocarpaceae & 1 & 19 \\
\hline Polygalaceae & 3 & 11 \\
\hline Primulaceae & 7 & 1095 \\
\hline Rhamnaceae & 1 & 1 \\
\hline Rosaceae & 3 & 24 \\
\hline Rubiaceae & 5 & 73 \\
\hline Smilacaceae & 1 & 9 \\
\hline Styracaceae & 1 & 6 \\
\hline Symplocaceae & 6 & 269 \\
\hline Total & 162 & 9578 \\
\hline
\end{tabular}




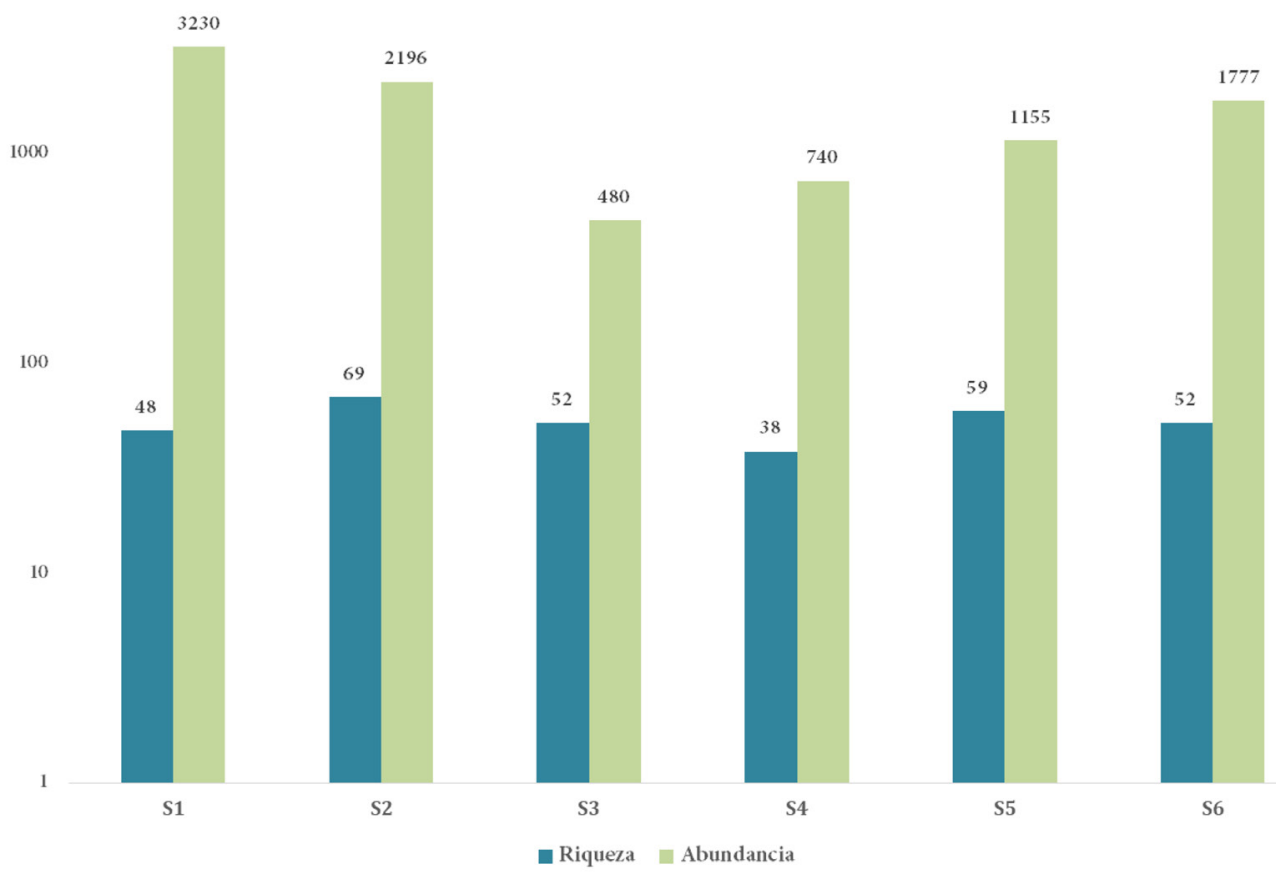

Figura 3. Riqueza y abundancia en los bosques esclerófilos de la Selva Central por cada sitio de muestreo.

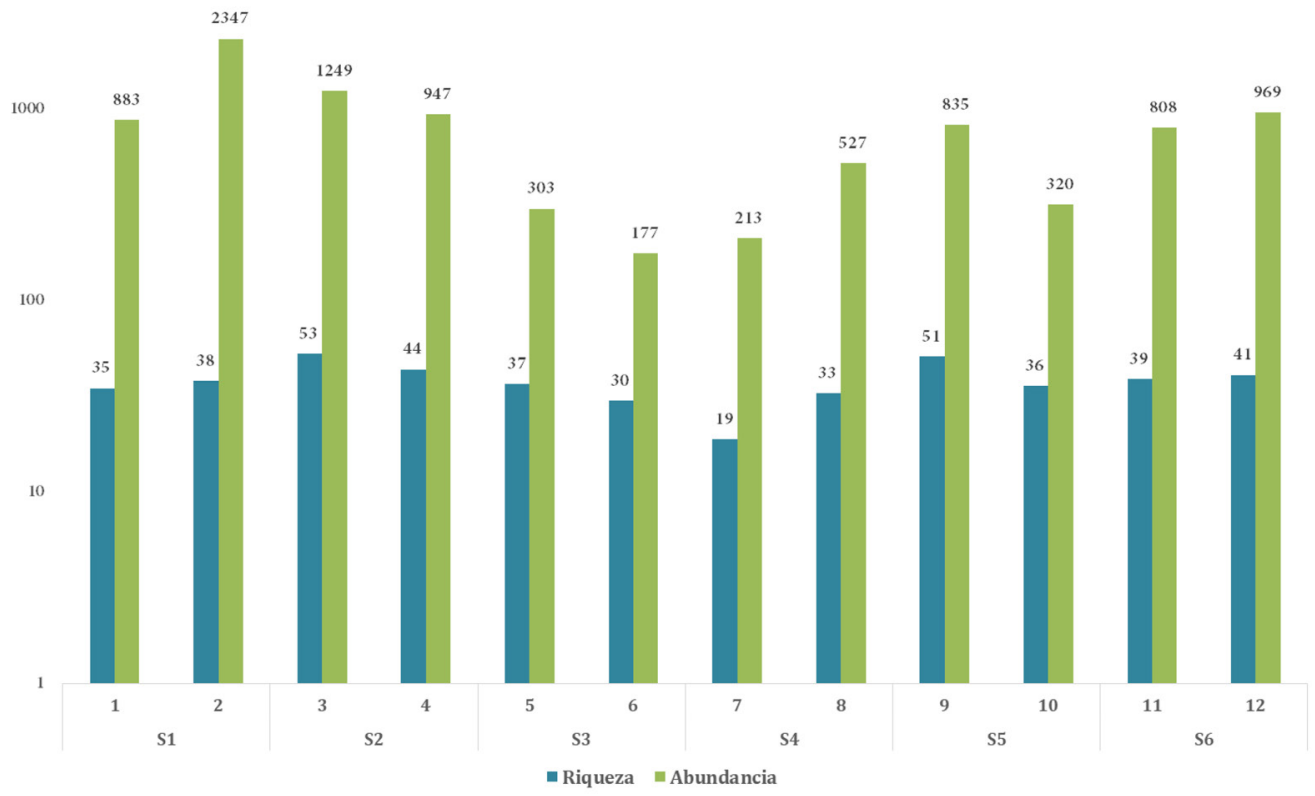

Figura 4. Riqueza y abundancia en los bosques esclerófilos de la Selva Central por cada parcela de muestreo. 
(parcela 2), frente a 883 registrados en la otra (parcela 1). En el caso de Milpo (S3), en una de las parcelas se registraron 177 individuos (parcela 6), mínima abundancia registrada a nivel de parcelas, mientras que, en la otra parcela 303 individuos (parcela 5). Además de los sitios ya mencionados, es importante remarcar el ACM Bosque Sho'llet (S5), donde la diferencia en el registro de abundancia entre las dos parcelas fue de 515 individuos.

A continuación se presenta un análisis detallado de la riqueza y abundancia por familias registrada en cada sitio de muestreo (ver Figura 5 y 6). En Oso Playa (S1), se registraron 48 especies distribuidas en 20 familias, de las cuales: Melastomataceae (8), Ericaceae (6), Aquifoliaceae (4), Clusiaceae (4), Asteraceae, Cunoniaceae, Penthaphylacae y Primulaceae, (3 spp. respectivamente), representaron el $70.83 \%$ del total de las especies. El resto de familias contienen una especie cada una. En cuanto a la abundancia, Clusiaceae fue registrada con 2071 (64.12\%) individuos, seguida por Primulaceae con 448 (13.87\%); ambas familias en conjunto congregaron el $77.99 \%$ de todos los individuos. Sin embargo, es importante mencionar que en una sola parcela se registraron 1832 individuos de Clusia sp.2 (Clusiaceae) que representó el $88.45 \%$ del total para la parcela 2.

En el Abra Esperanza (S2), se registraron 69 especies distribuidas en 24 familias, de las cuales: Asteraceae (8), Cunoniaceae (8), Melastomataceae (8), Ericaceae (7), Clusiaceae y Primulaceae (5 spp. respectivamente) congregaron el $59.42 \%$ de las especies. La vegetación más abundante estuvo representada principalmente por: Primulaceae con 389 individuos (17.70\%), seguida de Melastomataceae con 369 (16.79\%), Clusiaceae con 329 (14.97\%) y Asteraceae con 273 (12.42\%); las cuales representaron el $61.88 \%$.

En Milpo (S3), se registraron 52 especies distribuidas en 22 familias, de las cuales: Ericaceae (9), Melastomataceae (9), Asteraceae (5), Clusiaceae (3), Cunoniaceae y Primulaceae (3 spp. respectivamente) conformaron el $57.69 \%$ del total de las especies. La abundancia estuvo representada principalmente por: Ericaceae con
102 (21.25\%) individuos, Melastomataceae con 79 (16.46\%), Asteraceae con 60 (12.5\%), Pentaphylacaceae con 50 (10.42\%), y Cunoniaceae con 48 (10.00\%); en conjunto representaron el $70.63 \%$.

En San Daniel (S4), se registraron 38 especies distribuidas en 17 familias, de las cuales: Cunoniaceae (7), Ericaceae (6), Clusiaceae (4), Melastomataceae (4), Primulaceae y Asteraceae (3 spp. respectivamente) representaron el $71.05 \%$ del total de especies. El resto de familias contienen una especie cada una. La abundancia se encuentra representada principalmente por las familias: Clusiaceae con 422 (57.03\%), seguida de lejos por Cunoniaceae (8.24\%) con 61 individuos, ambas congregaron el $65.27 \%$.

En el ACM Bosque Sho'llet (S5), se registraron 59 especies distribuidas en 25 familias, de las cuales Cunoniaceae (8), Ericaceae (8), Clusiaceae (6), Melastomataceae (6), Aquifoliaceae y Asteraceae (4 spp. cada una) representaron el $61,02 \%$ del total de especies. La abundancia está conformada principalmente por: Chloranthaceae con 305 (26.41\%), Ericaceae $210(18.18 \%)$ y Clusiaceae $109(9.44 \%)$ y Melastomataceae 102 (8.83\%), que en conjunto representaron el $62.86 \%$.

En el Abra Yanachaga (S6), se registraron 52 especies distribuidas en 21 familias, de las cuales Ericaceae, Melastomataceae, Asteraceae (7 spp. cada una), Aquifoliaceae (5), Cunoniaceae y Symplocaceae (4 spp. cada una) contuvieron el $65.38 \%$ de total de especies. La abundancia estuvo conformada principalmente por las familias: Melastomataceae con 371 (20.88\%), Chloranthaceae con 316 (17,78\%), Symplocaceae con 233 (13.11\%), y Clusiaceae con 190 (10.69\%) individuos, que en conjunto congregaron el $62.46 \%$.

\section{Estructura de la vegetación}

Como ya se mencionó líneas arriba, se optó por evaluar individuos leñosos a partir de 1 $\mathrm{cm}$ de DAP, registrándose un total de 9578. Si el muestreo hubiera sido considerado a partir de $2.5 \mathrm{~cm}$ de DAP, los individuos registrados serían 2871, por lo tanto, solo se hubiera registrado el $29.97 \%$ del total registrado. El $70.03 \%$ 

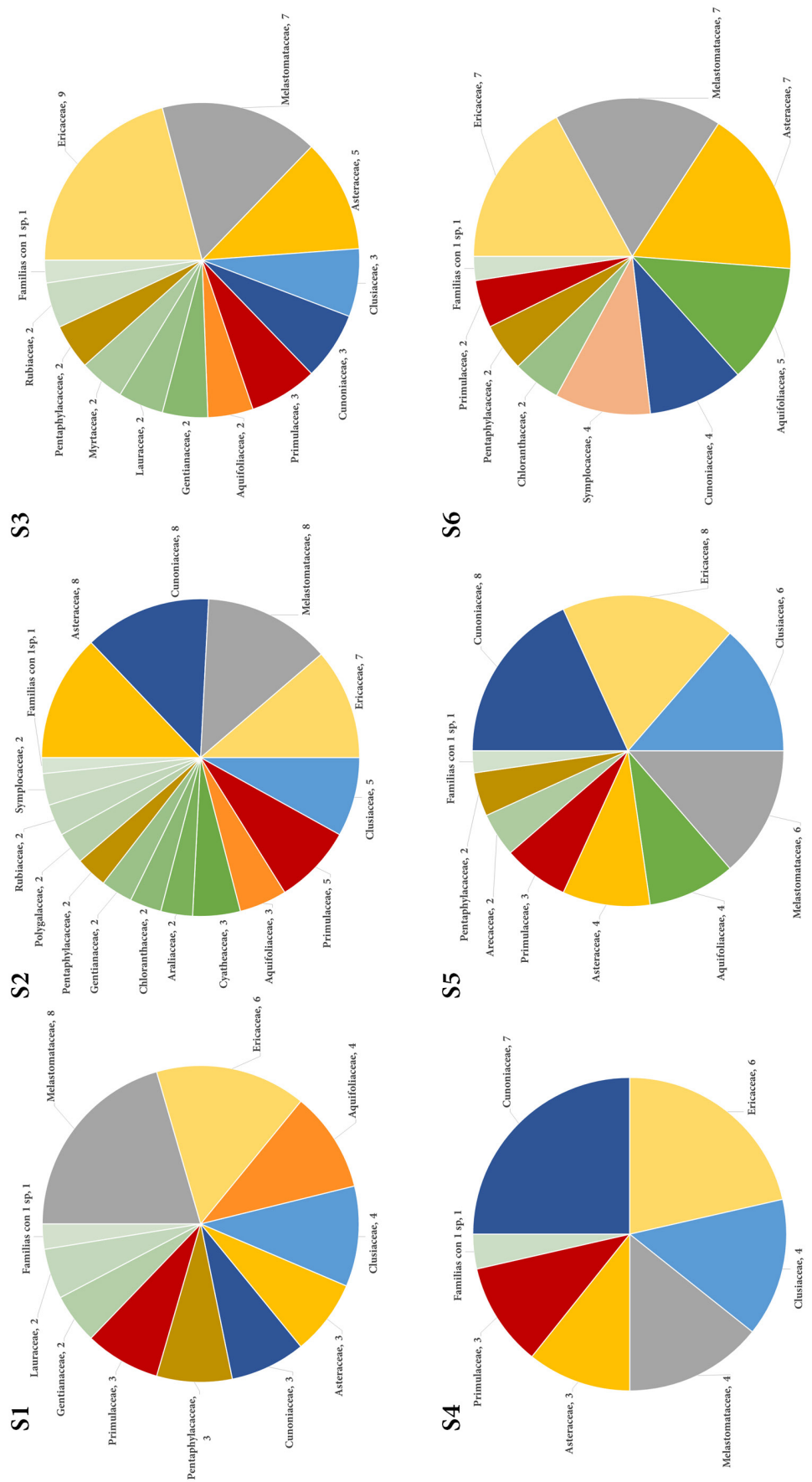

Figura 5. Riqueza de especies de los bosques esclerófilos por cada sitio de muestreo. 

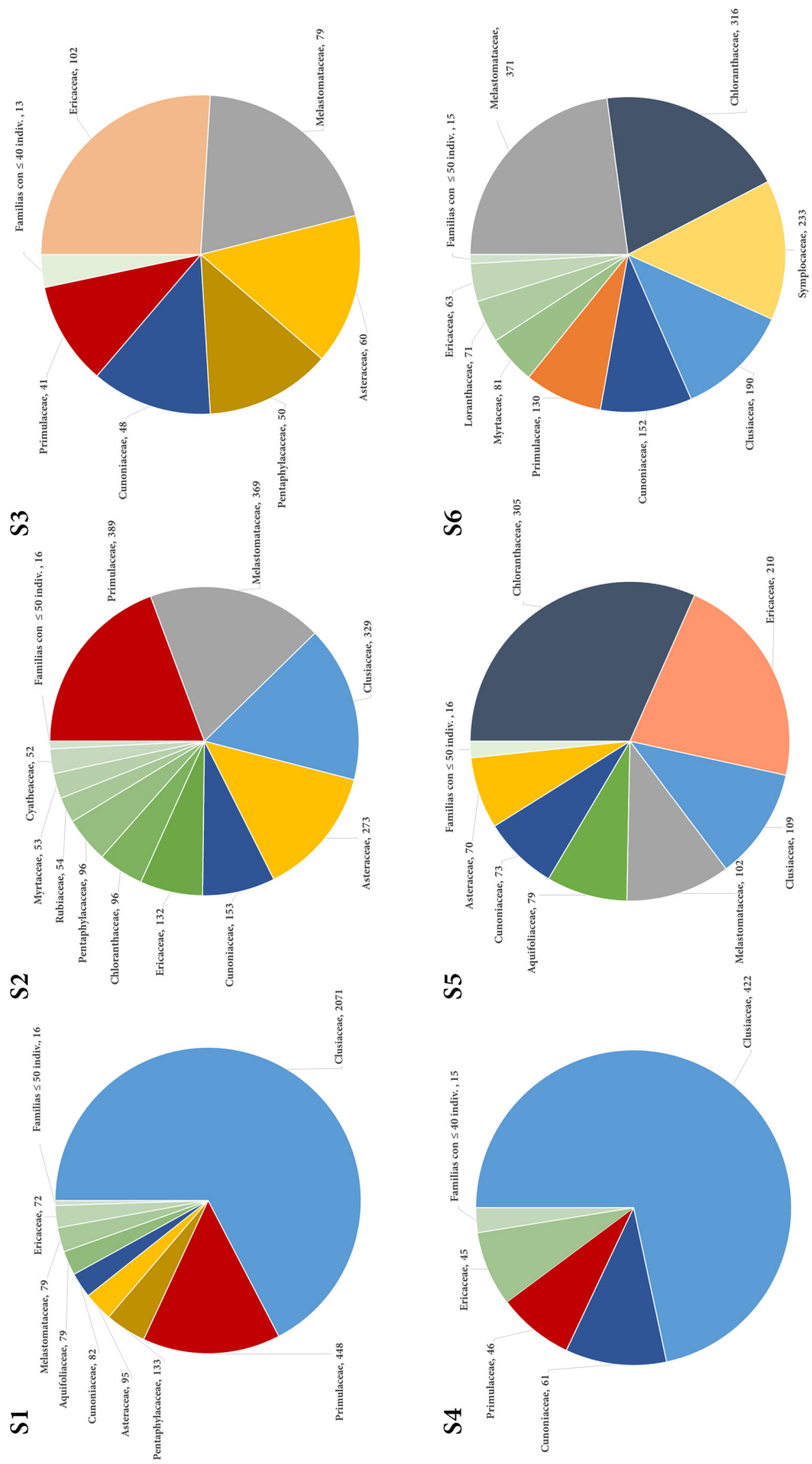

Figura 6. Abundancia de individuos de los bosques esclerófilos por cada sitio de muestreo. 

esclerófilos de la Selva Central del Perú

\begin{tabular}{|c|c|c|c|}
\hline $\begin{array}{c}\text { Clases Diamétricas } \\
(\mathbf{D A P} \text { cm) }\end{array}$ & No árboles & Porcentaje (\%) & $\begin{array}{c}\text { Número de árboles } \\
\text { por ha } \\
( \pm \mathbf{S D})\end{array}$ \\
\hline $1-1.99$ & 5295 & 55.28 & $4412.5( \pm 11.91)$ \\
\hline $2-4.99$ & 3529 & 36.84 & $2940.8( \pm 22.35)$ \\
\hline $5-9.99$ & 630 & 6.58 & $525( \pm 6.88)$ \\
\hline $10-19.99$ & 111 & 1.16 & $92.5( \pm 2.29)$ \\
\hline $20-29.99$ & 10 & 0.10 & $8.3( \pm 0.24)$ \\
\hline $30-40$ & 3 & 0.03 & $2.5( \pm 0.10)$ \\
\hline
\end{tabular}

Cuadro 4. Tamaño de clase para árboles y arbustos con $\geq 1 \mathrm{~cm}$ DAP en 1.2 ha en los bosques esclerófilos de la Selva Central.

de individuos registrados tuvieron un DAP que se distribuye entre $1-2.49 \mathrm{~cm}$, en un área de 1.2 ha.

Para mostrar claramente la estructura horizontal de estos bosques se ha realizado un análisis de agrupamiento por clases diamétricas, donde la mayor proporción de individuos se concentró entre $1-1.99 \mathrm{~cm}$, y representa el $55.28 \%$ del total (ver Cuadro 4).

De acuerdo al índice de valor de importancia (IVI), las 10 familias más importantes en los bosques esclerófilos fueron Clusiaceae (58.730), Chloranthaceae (25.095), Primulaceae (24.799), Cunoniaceae (23.224), Pentaphylacaceae (20.629), Melastomataceae (19.312), Ericaceae (18.957), Asteraceae (12.440), Aquifoliaceae (9.806) y Cyatheaceae (9.475) (ver Cuadro 5).

La familia Clusiaceae, con 58.730 de un total 300 , obtuvo la mayor abundancia basada principalmente en el sitio 1 (Oso Playa), en donde en una de las 2 parcelas se registraron 1832 individuos; este sitio estaba compuesto principalmente por arbustos de Clusia sp.2, los cuales no fueron encontrados en floración durante el periodo de evaluación. Además, es importante destacar que el género Clusia ha sido registrado en las 12 parcelas evaluadas.
Chloranthaceae, representada solo por 3 especies del género Hedyosmum; en términos de abundancia representó casi la cuarta parte de los individuos registrados en Clusiaceae, sin embargo, posee la segunda mayor área basal $\left(0.798 \mathrm{~m}^{2}\right)$, y fue registrado en 11 de 12 parcelas; por lo que tiene un IVI de 25.095, el segundo más alto registrado para estos bosques.

Primulaceae, a pesar de tener un tercio más de individuos que Chloranthaceae, tiene un área basal un tanto menor $\left(0.768 \mathrm{~m}^{2}\right)$ que la anterior, y fue registrada en las 12 parcelas, con un IVI de 24.799.

Pentaphylaceae, representada solo por tres especies (Ternstroemia meridionalis Mutis ex L. f. y 2 spp. de Freziera), y 336 individuos, tiene una de las áreas basales más alta $\left(0.621 \mathrm{~m}^{2}\right)$, lo que le ha permitido alcanzar un IVI de 20.629, y es la quinta familia de mayor importancia en los bosques esclerófilos.

Melastomataceae, fue registrada con 1024 individuos (tercera familia con mayor abundancia), sin embargo, su área basal es de 0.552 $\mathrm{m}^{2}$, lo que la convierte en la sexta familia en términos de IVI con 19.312.

En cuanto a la estructura vertical de estas comunidades vegetales, el $59.65 \%$ de los individuos se concentró en un rango de altura que

Cuadro 5. Abundancia, Área basal $\left(\mathrm{m}^{2}\right)$, Frecuencia e Índice de Valor de Importancia (IVI) para las familias registradas en los bosques esclerófilos de la Selva Central (Cuadro en pagína siguiente). 


\begin{tabular}{|c|c|c|c|c|c|c|c|}
\hline Familia & Abundancia & $(\%)$ & Área Basal & $(\%)$ & Frecuencia & $(\%)$ & IVI \\
\hline Clusiaceae & 3128 & 26.698 & 2.108 & 26.698 & 12 & 5.333 & 58.730 \\
\hline Chloranthaceae & 764 & 10.103 & 0.798 & 10.103 & 11 & 4.889 & 25.095 \\
\hline Primulaceae & 1095 & 9.733 & 0.768 & 9.733 & 12 & 5.333 & 24.799 \\
\hline Cunoniaceae & 569 & 8.945 & 0.706 & 8.945 & 12 & 5.333 & 23.224 \\
\hline Pentaphylacaceae & 336 & 7.870 & 0.621 & 7.870 & 11 & 4.889 & 20.629 \\
\hline Melastomataceae & 1024 & 6.989 & 0.552 & 6.989 & 12 & 5.333 & 19.312 \\
\hline Ericaceae & 624 & 6.812 & 0.538 & 6.812 & 12 & 5.333 & 18.957 \\
\hline Asteraceae & 570 & 3.553 & 0.281 & 3.553 & 12 & 5.333 & 12.440 \\
\hline Aquifoliaceae & 270 & 2.458 & 0.194 & 2.458 & 11 & 4.889 & 9.806 \\
\hline Cyatheaceae & 68 & 3.404 & 0.269 & 3.404 & 6 & 2.667 & 9.475 \\
\hline Lauraceae & 99 & 2.727 & 0.215 & 2.727 & 8 & 3.556 & 9.010 \\
\hline Podocarpaceae & 19 & 3.017 & 0.238 & 3.017 & 6 & 2.667 & 8.700 \\
\hline Loranthaceae & 188 & 1.473 & 0.116 & 1.473 & 12 & 5.333 & 8.278 \\
\hline Symplocaceae & 269 & 1.922 & 0.152 & 1.922 & 6 & 2.667 & 6.510 \\
\hline Gentianaceae & 42 & 0.628 & 0.050 & 0.628 & 9 & 4.000 & 5.256 \\
\hline Araliaceae & 62 & 0.564 & 0.045 & 0.564 & 8 & 3.556 & 4.684 \\
\hline Rubiaceae & 73 & 0.206 & 0.016 & 0.206 & 9 & 4.000 & 4.413 \\
\hline Myrtaceae & 151 & 0.367 & 0.029 & 0.367 & 8 & 3.556 & 4.289 \\
\hline Clethraceae & 58 & 0.907 & 0.072 & 0.907 & 5 & 2.222 & 4.037 \\
\hline Alzateaceae & 37 & 0.550 & 0.043 & 0.550 & 4 & 1.778 & 2.877 \\
\hline Rosaceae & 24 & 0.076 & 0.006 & 0.076 & 5 & 2.222 & 2.374 \\
\hline Polygalaceae & 11 & 0.040 & 0.003 & 0.040 & 5 & 2.222 & 2.303 \\
\hline Brunelliaceae & 22 & 0.228 & 0.018 & 0.228 & 4 & 1.778 & 2.235 \\
\hline Arecaceae & 11 & 0.354 & 0.028 & 0.354 & 3 & 1.333 & 2.042 \\
\hline Columelliaceae & 21 & 0.129 & 0.010 & 0.129 & 4 & 1.778 & 2.036 \\
\hline Campanulaceae & 8 & 0.036 & 0.003 & 0.036 & 4 & 1.778 & 1.850 \\
\hline Adoxaceae & 10 & 0.041 & 0.003 & 0.041 & 3 & 1.333 & 1.416 \\
\hline Styracaceae & 6 & 0.057 & 0.004 & 0.057 & 2 & 0.889 & 1.003 \\
\hline Smilacaceae & 9 & 0.021 & 0.002 & 0.021 & 2 & 0.889 & 0.931 \\
\hline Hypericaceae & 2 & 0.004 & 0.000 & 0.004 & 2 & 0.889 & 0.896 \\
\hline Culcitaceae & 1 & 0.043 & 0.003 & 0.043 & 1 & 0.444 & 0.531 \\
\hline Berberidaceae & 3 & 0.024 & 0.002 & 0.024 & 1 & 0.444 & 0.492 \\
\hline Caprifoliaceae & 2 & 0.011 & 0.001 & 0.011 & 1 & 0.444 & 0.465 \\
\hline Myricaceae & 1 & 0.005 & 0.0004 & 0.005 & 1 & 0.444 & 0.453 \\
\hline Rhamnaceae & 1 & 0.004 & 0.0003 & 0.004 & 1 & 0.444 & 0.452 \\
\hline Total & 9578 & 100 & 7.894 & 100 & 225 & 100 & 300 \\
\hline
\end{tabular}



esclerófilos de la Selva Central del Perú

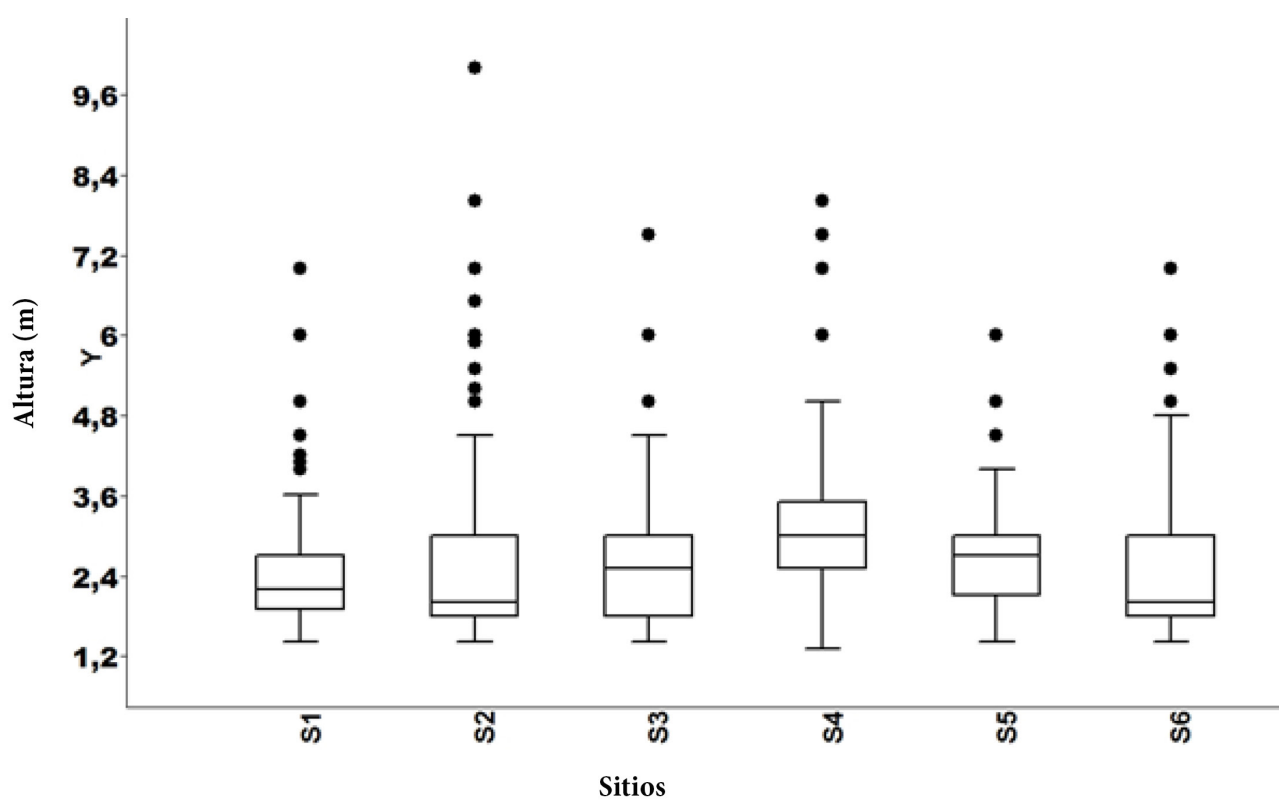

Figura 7. Estructura vertical de la vegetación leñosa en los bosques esclerófilos de la Selva Central.

va entre los 2-3 m (ver Figura 7). Sin embargo, fueron registrados individuos que están por encima de este rango, el $13.48 \%$ de individuos se encontraron con una altura $>3$ hasta $5 \mathrm{~m}$, y el 1.01\% (97 individuos) fueron registrados con una altura $>5 \mathrm{~m}$, de los cuales un solo individuo tenía $10 \mathrm{~m}$ (máxima altura registrada).

\section{Análisis de ordenación}

Se hizo este análisis en base al Escalamiento Multidimensional No Métrico para las 12 parcelas de muestreo de los seis sitios de evaluación utilizando el índice de similitud de Morisita-Horn, en donde el Stress que se obtuvo fue de 0.1763 , luego de una corrida de los datos, pues el Stress se mantuvo constante durante las siguientes corridas de datos. El valor del Strees nos indica que la distribución de las muestras fue buena.

Este análisis reflejó un bajo grado de afinidad entre los seis sitios evaluados. Sin embargo, se encontró una alta similitud entre las parcelas de tres sitios: San Daniel (S4), ACM Bosque Sho'llet (S5) y Abra Yanachaga (S6). Las parcelas de los tres sitios restantes presentaron una relativa similitud (ver líneas punteadas en verde, Figura 8). Asimismo, la parcela 2 de Oso Playa (S1-P2) fue la que menor afinidad ha registrado respecto al resto de parcelas evaluadas, pues tiene una densidad de individuos compuesta principalmente por individuos de Clusia sp.2.

\section{Discusión}

Se han realizado muy pocos estudios en bosque esclerófilo o bosque achaparrado, la gran parte de la bibliografía encontrada se enfoca en reportes de composición florística y descripción del área mas no en la estructura de la vegetación. Este tipo de vegetación con similares características en términos de estructura ha sido reportada en la Cordillera del Cóndor en Ecuador (Jadán y Aguirre 2013), en los tepuis de Venezuela (Steyermark et al. 1995) y en los bosques de Luquillo en Puerto Rico (Weaver et al. 1986). Asimismo, en el Perú existen reportes de bosques esclerófilos, como los registrados en la cuenca del Río Cenepa (Vásquez 2010) y en la Reserva Comunal El Sira (Valenzuela 2015). 


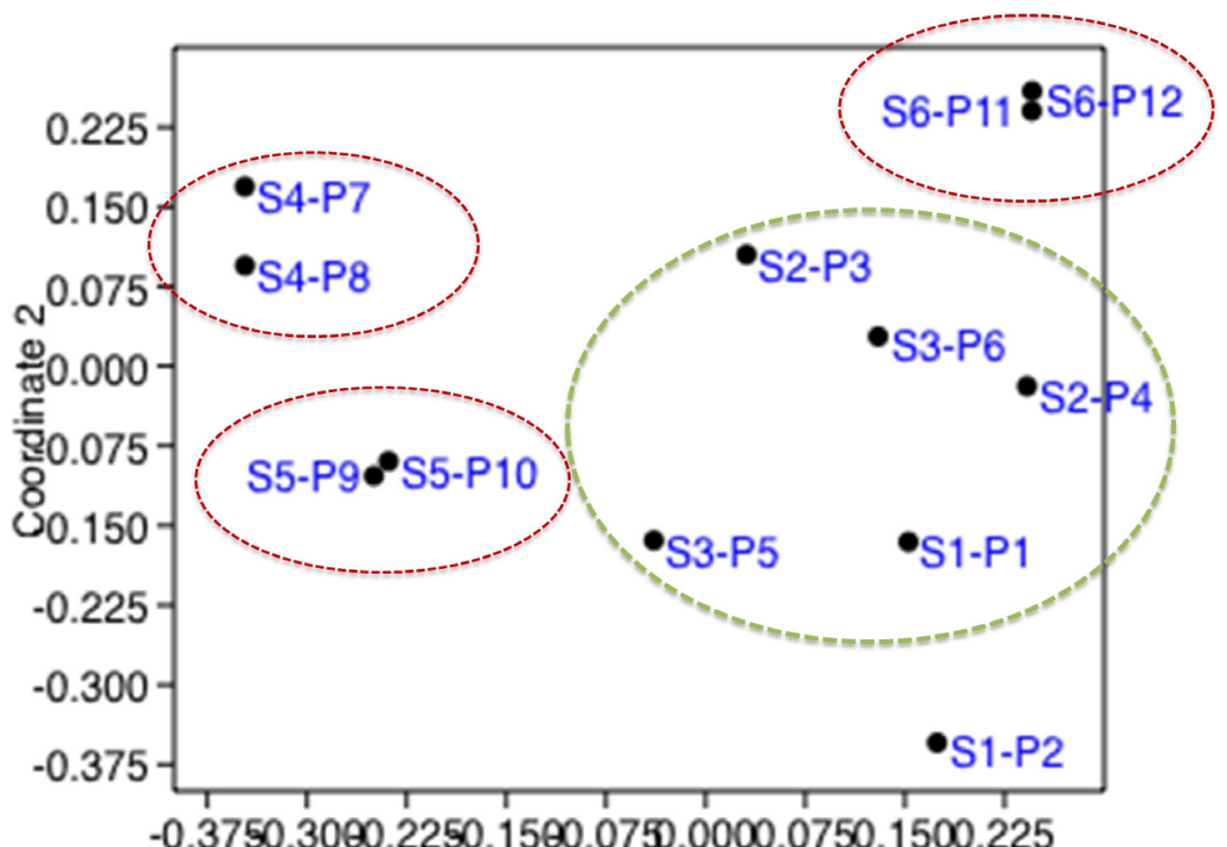

Coordinate 1

Figura 8. Análisis de ordenación de las 12 parcelas de los seis sitios de muestro (Stress: 0.1763).

Se registraron 162 especies leñosas en 1.2 ha $\left(12000 \mathrm{~m}^{2}\right)$ distribuidas en seis sitios dentro y fuera del PNYCh y el ACM Bosque Sho'llet. Uno de los sitios evaluados (Milpo - S3) claramente estaba perturbado por acciones antrópicas de tala y quema de vegetación, este sitio se encuentra sin protección ya que está ubicado fuera del PNYCh, así como de la zona de amortiguamiento (ver Figura 1); a diferencia de este, los demás sitios se encontraron sin señales recientes de perturbación por acción humana.

En el estudio de Jadán y Aguirre (2013) en la Cordillera del Cóndor donde denominan a este tipo de vegetación como "bosque chaparro", se determinaron 55 especies de arbustos en un área de $300 \mathrm{~m}^{2}$ y 76 especies de árboles en $2400 \mathrm{~m}^{2}$ (reporte al 50\% de la identificación de muestras colectadas). Una de las principales razones en diferencias de la riqueza puede recaer en el tamaño muestral, así como, en la fal- ta de identificación de muestras en este último estudio.

Sobre la riqueza de especies a nivel de familias, Melastomataceae (21) y Asteraceae (19) son las más diversas de estos bosques, lo cual coincide con el reporte de Vásquez (2005), donde menciona a estas familias entre las 15 con mayor contribución en número de especies a la flora vascular de la Selva Central. Estas dos familias junto a Ericaceae (18), Cunoniaceae (16), Aquifoliaceae (12), Clusiaceae (9), Primulaceae (7), Symplocaceae (6), Rubiaceae (5), y Pentaphylaceae (4) contienen más del $70 \%$ de la riqueza de especies. Mientras que, en la Cordillera del Cóndor para este tipo de vegetación se encontró que las familias con mayor riqueza de especies fueron Clusiaceae (8), Melastomataceae (7) y Lauraceae (6) (Jadán y Aguirre 2013), aunque con claras diferencias en cuanto al número de especies. En cuanto a la abundancia de individuos, Clusiaceae fue la familia con la mayor cantidad de individuos 

esclerófilos de la Selva Central del Perú

muy por encima de las otras familias, pues representó casi un tercio del total de individuos registrados en los seis sitios. Sin embargo, esta abundancia está altamente influenciada por la dominancia de arbustos de Clusia sp.2 en una de las parcelas en Oso Playa (S1), donde el $88.45 \%$ de individuos pertenecían a esta morfoespecie.

Tanto la riqueza como la abundancia a nivel de familias varía ampliamente entre los sitios de muestreo (ver Figura 5 y 6), e incluso entre las parcelas de un mismo sitio (ver Figura 4), sin embargo, se ha encontrado que ciertas familias, aunque con distintas riquezas y abundancias son predominantemente recurrentes en los sitios de muestreo, por ejemplo: $\mathrm{Me}$ lastomataceae y Ericaceae contienen una de las mayores riquezas de especies para los seis sitios, mientras que, Clusiaceae fue registrada con una de las mayores riquezas para cinco sitios. Para el caso de la abundancia, un claro ejemplo es Clusiaceae, que fue encontrada como la más abundante en Oso Playa (S1), y en San Daniel (S4), con 2071 y 422 individuos respectivamente.

Sobre la estructura diamétrica de la vegetación, se puede corroborar que el $92.12 \%$ de los individuos en estos bosques tienen menos de $5 \mathrm{~cm}$ de diámetro. De otro lado, el IVI encontrado por familia indica que no hay una familia con representatividad absoluta, sin embargo, Clusiaceae (58.730) tiene un valor que es el doble de la subsiguiente familia Chloranthaceae (25.095), esto debido al área basal de $2.108 \mathrm{~m}^{2}$ de la primera familia frente a $0.798 \mathrm{~m}^{2}$ de la segunda. El área basal de Clusiaceae está influenciado por la alta densidad de individuos registrados para esta familia como ya ha sido ha sido explicado anteriormente. La estructura vertical para estos bosques fue registrada entre los 2-3 m de alto en casi el $60 \%$ de los individuos. Estos datos de estructura permiten tener un claro conocimiento de cómo se desarrolla esta vegetación. Finalmente, la similitud entre los sitios de evaluación es baja, pues cada sitio tiene una riqueza y abundancia con características particulares. El distribución de este tipo de vegetación en los sitios evaluados generalmente es a modo de parches entremez- clados con pajonales húmedos, dichos parches tienen diferentes tamaños, lo cual también influye en la abundancia y riqueza de especies.

Los factores abióticos están jugando un rol fundamental en la composición y estructura de los bosques esclerófilos, principalmente, la altitud, los suelos, la precipitación, las pendientes, y los vientos influyen directamente en la forma de crecimiento de esta vegetación. Debido a ello son necesarios estudios de este tipo para entender mejor la dinámica de esta vegetación.

\section{Conclusiones}

Se ha determinado que la composición florística de la vegetación leñosa de los bosques esclerófilos evaluados está conformada por 35 familias, 61 géneros y 162 especies de plantas vasculares.

Con respecto a la estructura, la distribución diamétrica de la vegetación leñosa se concentra entre 1-4.99 cm de diámetro, en el $92.12 \%$ de los individuos. Mientras que la distribución altimétrica tiene un rango de $2-3 \mathrm{~m}$ en el $59.65 \%$, y de $3-5 \mathrm{~m}$ en el $13.48 \%$ de individuos.

Las diez familias con mayor importancia en términos de IVI fueron Clusiaceae (58.730), Chloranthaceae (25.095), Primulaceae (24.799), Cunoniaceae (23.224), Pentaphylacaceae (20.629), Melastomataceae (19.312), Ericaceae (18.957), Asteraceae (12.440), Aquifoliaceae (9.806) y Cyatheaceae (9.475).

Hay un bajo grado de similitud entre los seis sitios evaluados, pero un alto grado de afinidad entre las parcelas de los sitios: San Daniel (S4), ACM Sho'llet (S5) y Abra Yanchaga (S6). Además, la parcela 2 de Oso playa (S1-P2) presentó la menor afinidad respecto al resto.

\section{Agradecimientos}

Al Jardín Botánico de Missouri por haber brindado los fondos para el desarrollo de esta investigación, y por todas las facilidades otorgadas durante el desarrollo del proyecto. Asimismo, al Herbario HOXA y USM por otorgar la permisos de ingreso para la identificación de los especímenes; a la Municipalidad de 
Oxapampa por la autorización para el ingreso al Área de Conservación Municipal Bosque Sho'llet. Finalmente, la autora agradece a todas las personas que contribuyeron durante el trabajo de campo, así como, a quienes apoyaron durante la redacción del manuscrito.

\section{Bibliografía}

APG IV, (Angiosperm Phylogeny Group); Chase, MW; Christenhusz, MJM; Fay, MF; Byng, JW; Judd, WS; Soltis, DE; Mabberley, DJ; Sennikov, AN; Soltis, PS; Stevens, PF; Briggs, B; Brockington, S; Chautems, A; Clark, JC; Conran, J; Haston, E; Möller, M; Moore, M; Olmstead, R; Perret, M; Skog, L; Smith, J; Tank, D; Vorontsova, M; Weber, A. 2016. An update of the Angiosperm Phylogeny Group classification for the orders and families of flowering plants: APG IV. Botanical Journal of the Linnean Society 181(1):1-20.

Campbell, P; Comiskey, J; Alonso, A; Dallmeier, F; Nuñez, P; Beltran, P; Baldeon, S; Nauray, W; De la Colina, R; Acurio, L; Udvardy, S. 2002. Modified Whittaker plots as an assessment and monitoring tool for vegetation in a lowland tropical rainforest. Environmental Monitoring and Assessment. Kluwer Academic Publishers. USA. 76: 19-41 pp.

Colwell RK; Coddington JA. 1994. Estimating terrestrial biodiversity through extrapolation. Philosophical Transections of the Royal Society of London. Series B: Biological Sciences 345(1311):101-118.

Colwell, RK. 2013. EstimateS: Statistical estimation of species richness and shared species from samples. V. 9.1.0. User's Guide and application.

Font Quer, P. 1953. Diccionario de Botánica. Ediciones Península. s. a. Peu de la Creu 4, 08001- Barcelona, España.

Hammer, Ø. 1999-2018. PAST. PAleontological STatistics. Version 3.22. Reference manual. Natural History Museum. University of Oslo.

Hammer, Ø; Harper, DAT; Ryan, PD. 2001. PAST: Paleontological statistics software pack- age for education and data analysis. Palaeontologia Electronica 4(1):1-9.

Jadán, O; Aguirre Mendoza, Z. 2013. Capítulo 2: Flora de los Tepuyes de la Cuenca Alta del Río Nangaritza, Cordillera del Cóndor en Evaluación Ecológica Rápida de la Biodiversidad de los Tepuyes de la Cuenca Alta del Río Nangaritza, Cordillera Del Cóndor, Ecuador.

Magurran, EA. 2004. Measuring Biological Diversity. Blackwell Publishing, Oxford, 256 p.

Palmer, MW. 1990. The stimation of species richness by extrapolation. Ecology 71:11951198.

ProNaturaleza. s/f. Área de Conservación $\mathrm{Mu}-$ nicipal "El Bosque del Sho'llet" (Documento de trabajo). Expediente técnico justificatorio. Villa Rica-Oxapampa-Perú. Pro Naturaleza-PSC -Recursos Naturales.

Philip, O; Baker, T. 2002-2006. Manual de campo para el establecimiento y remedición de parcelas permanentes - RAINFOR. PAN-AMAZONÍA: Project the Advancement of Networked Science in Amazonia.

Sevicio Nacional de Áreas Naturales Protegidas por el Estado (SERNANP). 2015. Plan Maestro del Parque Nacional Yanachaga Chemillén 2015-2019. Lima, Perú. Dirección de Desarrollo Estratégico del Sevicio Nacional de Áreas Naturales Protegidas por el Estado. 76 p.

Steyermark, JA; Berry, PE; Yatskievych, K; Holst, BK. (ed.). 1995. The Flora of the Venezuelan Guayana. Vol. 1. Missouri Botanical Garden y Timber Press, St. Louis, Missouri. 320 p.

Valenzuela, L; Vásquez, R; Rojas, R; Villalba, MI; Phillips, O; López, G; Chama, V; Monteagudo, A; Bellota, D; Huillca, Y; Pallqui, NC. 2015. Línea base para el monitoreo de la vegetación en la Reserva Comunal El Sira (RCS). Arnaldoa 22: 243-268.

Vasquez, R; Rojas, R; Monteagudo, A.; Meza, K.; Van Der Werff, H. \& Catchpole, D. 2005. Flora Vascular de la Selva Central del Perú: Una aproximación de la composición florística de tres Áreas Naturales Protegidas. Arnaldoa 12:112-125. 
Vásquez, R; Rojas, R; Van der Werff, H. 2010. Flora del Río Cenepa, Amazonas, Perú. Vol. 1. Missouri Botanical Garden Press. 1568 p.

Villarreal, H; Álvarez, M; Córdoba, S; Escobar, F; Fagua, G; Gast, F; Mendoza, H; Ospina, M; Umaña, AM. 2006. Manual de Métodos para el Desarrollo de Inventarios de Biodiversidad. Segunda edición. Programa Inventarios de Biodiversidad. Grupo de Exploración y Monitoreo Ambiental (GEMA). Instituto de Investigación de Recursos Biológicos Alexander von Humboldt. Alexander von Humboldt. Bogotá, Colombia. 236 p.

Weaver, PL; Medina, E; Pool, D; Dugger, K; Gonzales-Liboy, J; Cuevas, E; 1986. Ecological Observations in the Dwarf Cloud Forest of the Luquillo Mountains in Puerto Rico. Biotropica 18(1):79-85.

Presentado: 21/11/2018

Aceptado: 28/12/2018

Publicado en línea: 28/12/2018 


\section{Anexos}

Anexo 1. Composición florística de la vegetación leñosa de los bosques esclerófilos del PNYCH, ACM Sho'llet y zonas aledañas (Nota: $1=$ presencia; $0=$ ausencia).

\begin{tabular}{|c|c|c|c|c|c|c|}
\hline Familia/Especie & S1 & S2 & S3 & S4 & S5 & S6 \\
\hline Adoxaceae & $\mathbf{0}$ & $\mathbf{0}$ & $\mathbf{0}$ & 1 & 1 & $\mathbf{0}$ \\
\hline Viburnum hallii (Oerst.) Killip \& A. C. Sm. & 0 & 0 & 0 & 1 & 0 & 0 \\
\hline Viburnum sp. 1 & 0 & 0 & 0 & 0 & 1 & 0 \\
\hline Alzateaceae & $\mathbf{0}$ & $\mathbf{0}$ & $\mathbf{0}$ & 1 & 1 & $\mathbf{0}$ \\
\hline Alzatea verticillata Ruiz \& Pav. & 0 & 0 & 0 & 1 & 1 & 0 \\
\hline Aquifoliaceae & 1 & 1 & 1 & 1 & 1 & 1 \\
\hline Ilex crassifolioides Loes. & 0 & 0 & 0 & 0 & 0 & 1 \\
\hline Ilex ericoides Loes. & 0 & 1 & 0 & 0 & 1 & 0 \\
\hline Ilex hualgayoca Loizeau \& Spichiger & 0 & 0 & 0 & 0 & 1 & $\mathbf{1}$ \\
\hline Ilex laureola Triana & 1 & 0 & 0 & 0 & 1 & 0 \\
\hline Ilex ovalis (Ruiz \& Pavon) Loesener & 1 & 0 & 0 & 0 & 0 & 0 \\
\hline Ilex sp.1 & 0 & 0 & 0 & 1 & 0 & 0 \\
\hline Ilex sp.2 & 1 & 0 & 1 & 0 & 0 & 0 \\
\hline Ilex sp. 3 & 1 & 1 & 0 & 0 & 0 & 1 \\
\hline Ilex sp.4 & 0 & 0 & 0 & 0 & 0 & 1 \\
\hline Ilex sp. 5 & 0 & 0 & 1 & 0 & 0 & 1 \\
\hline Ilex sp.6 & 0 & 0 & 0 & 0 & 1 & 0 \\
\hline Ilex suprema Cuatrec. & 0 & 1 & 0 & 0 & 0 & 0 \\
\hline Araliaceae & 1 & 1 & 1 & $\mathbf{0}$ & 1 & 1 \\
\hline Schefflera angulata (Pav.) Harms & 0 & 1 & 0 & 0 & 1 & 0 \\
\hline Schefflera pedicellata (Ruiz \& Pav.) Harms & 0 & 0 & 0 & 0 & 0 & 1 \\
\hline Schefflera sp.1 & 1 & 1 & 1 & 0 & 0 & 0 \\
\hline Arecaceae & 1 & $\mathbf{0}$ & $\mathbf{0}$ & $\mathbf{0}$ & 1 & $\mathbf{0}$ \\
\hline Ceroxylum vogelianum (Engel.) H. Wendl. & 0 & 0 & 0 & 0 & 1 & 0 \\
\hline Geonoma & 1 & $\mathbf{0}$ & $\mathbf{0}$ & $\mathbf{0}$ & 1 & $\mathbf{0}$ \\
\hline Geonoma aff. weberbaueri Dammer ex Burret. & 0 & 0 & 0 & 0 & 1 & 0 \\
\hline Geonoma sp.1 & 1 & 0 & 0 & 0 & 0 & 0 \\
\hline Asteraceae & 1 & 1 & 1 & 1 & 1 & 1 \\
\hline AST. sp.1 & 0 & 1 & 0 & 0 & 0 & 0 \\
\hline Baccharis nitida (Ruiz \& Pav.) Pers. & 0 & 0 & 1 & 1 & 1 & 0 \\
\hline Baccharis oblongifolia (Ruiz \& Pav.) Pers. & 1 & 1 & 1 & 1 & 1 & 0 \\
\hline
\end{tabular}




\begin{tabular}{|c|c|c|c|c|c|c|}
\hline Familia/Especie & S1 & S2 & S3 & S4 & S5 & S6 \\
\hline Asteraceae & 1 & 1 & 1 & 1 & 1 & 1 \\
\hline Baccharis sp.1 & 0 & 0 & 0 & 0 & 1 & 0 \\
\hline Baccharis sp. 2 & 0 & 0 & 0 & 0 & 0 & 1 \\
\hline Diplostephium vermiculatum Cuatrec. & 1 & 1 & 1 & 0 & 0 & 1 \\
\hline Gynoxys sp.1 & 0 & 0 & 1 & 0 & 0 & 0 \\
\hline Gynoxys sp.2 & 0 & 0 & 0 & 0 & 0 & 1 \\
\hline Gynoxys sp.3 & 0 & 0 & 0 & 0 & 0 & 1 \\
\hline Loricaria thuyoides var. stuebelii (Hieron) Cuatrec. & 0 & 0 & 0 & 0 & 0 & 1 \\
\hline Mikania sp. 2 & 1 & 0 & 0 & 0 & 0 & 0 \\
\hline Mikania sp.3 & 0 & 1 & 0 & 0 & 0 & 0 \\
\hline Mikania sp.4 & 0 & 1 & 0 & 0 & 0 & 0 \\
\hline Mikania szyszylowiczii Hieron. & 0 & 1 & 0 & 0 & 0 & 0 \\
\hline Munnozia sp.2 & 0 & 0 & 0 & 1 & 0 & 0 \\
\hline Munnozia sp.4 & 0 & 0 & 0 & 0 & 0 & 1 \\
\hline Nordenstamia tovarii (H. Rob. \& Cuatrec.) B. Nord. & 0 & 1 & 0 & 0 & 1 & 1 \\
\hline Pentacalia sp.2 & 0 & 0 & 1 & 0 & 0 & 0 \\
\hline Pentacalia $\mathrm{sp} .3$ & 0 & 1 & 0 & 0 & 0 & 0 \\
\hline Berberidaceae & $\mathbf{0}$ & $\mathbf{0}$ & $\mathbf{0}$ & $\mathbf{0}$ & 1 & $\mathbf{0}$ \\
\hline Berberis sp.1 & 0 & 0 & 0 & 0 & 1 & 0 \\
\hline Brunelliaceae & 1 & $\mathbf{0}$ & $\mathbf{0}$ & $\mathbf{0}$ & 1 & $\mathbf{0}$ \\
\hline Brunellia sp.1 & 0 & 0 & 0 & 0 & 1 & 0 \\
\hline Brunellia sp.2 & 1 & 0 & 0 & 0 & 0 & 0 \\
\hline Campanulaceae & 1 & 1 & 1 & $\mathbf{0}$ & $\mathbf{0}$ & $\mathbf{0}$ \\
\hline Siphocampylus pavonis E. Wimm. & 1 & 1 & 1 & 0 & 0 & 0 \\
\hline Caprifoliaceae & $\mathbf{0}$ & $\mathbf{0}$ & $\mathbf{0}$ & 1 & $\mathbf{0}$ & $\mathbf{0}$ \\
\hline Valeriana $\mathrm{sp} .2$ & 0 & 0 & 0 & 1 & 0 & 0 \\
\hline Chloranthaceae & 1 & 1 & 1 & 1 & 1 & 1 \\
\hline Hedyosmum cuatrecazanum Occhioni & 0 & 0 & 0 & 1 & 1 & 0 \\
\hline Hedyosmum cumbalense H. Karst. & 0 & 1 & 0 & 0 & 0 & 1 \\
\hline Hedyosmum dombeyanum Solms & 1 & 1 & 1 & 0 & 0 & 1 \\
\hline Clethraceae & $\mathbf{0}$ & $\mathbf{0}$ & 1 & 1 & 1 & $\mathbf{0}$ \\
\hline Clethra revoluta (Ruiz \& Pav.) Spreng. & 0 & 0 & 1 & 1 & 1 & 0 \\
\hline Clusiaceae & 1 & 1 & 1 & 1 & 1 & 1 \\
\hline Clusia multiflora Kunth & 1 & 1 & 1 & 1 & 1 & 1 \\
\hline
\end{tabular}




\begin{tabular}{|c|c|c|c|c|c|c|}
\hline Familia/Especie & S1 & S2 & S3 & S4 & S5 & S6 \\
\hline Clusiaceae & 1 & 1 & 1 & 1 & 1 & 1 \\
\hline Clusia sp.1 & 1 & 1 & 0 & 0 & 1 & 0 \\
\hline Clusia sp.2 & 1 & 0 & 1 & 0 & 1 & 0 \\
\hline Clusia sp.3 & 0 & 0 & 0 & 1 & 0 & 0 \\
\hline Clusia sp.4 & 1 & 0 & 0 & 0 & 1 & 0 \\
\hline Clusia sp.5 & 0 & 1 & 0 & 1 & 0 & 0 \\
\hline Clusia sp.6 & 0 & 0 & 1 & 0 & 1 & 0 \\
\hline Clusia sp.7 & 0 & 1 & 0 & 1 & 1 & 0 \\
\hline Clusia sp.8 & 0 & 1 & 0 & 0 & 0 & 0 \\
\hline Columelliaceae & $\mathbf{0}$ & 1 & 1 & $\mathbf{0}$ & $\mathbf{0}$ & 1 \\
\hline Desfontainia spinosa Ruiz \& Pav. & 0 & 1 & 1 & 0 & 0 & 1 \\
\hline Culcitaceae & $\mathbf{0}$ & 1 & $\mathbf{0}$ & $\mathbf{0}$ & $\mathbf{0}$ & $\mathbf{0}$ \\
\hline Culcita coniifolia (Hook.) Makon & 0 & 1 & 0 & 0 & 0 & 0 \\
\hline Cunoniaceae & 1 & 1 & 1 & 1 & 1 & 1 \\
\hline Weinmannia aff. pinnata $\mathrm{L}$. & 0 & 0 & 0 & 0 & 1 & 1 \\
\hline Weinmannia balbisiana Kunth & 1 & 0 & 0 & 1 & 1 & 0 \\
\hline Weinmannia cochensis Hieron. & 0 & 1 & 0 & 0 & 0 & 0 \\
\hline Weinmannia glabra L. f. & 0 & 1 & 0 & 0 & 0 & 0 \\
\hline Weinmannia jelskii Szyszyl. & 1 & 1 & 1 & 0 & 1 & 1 \\
\hline Weinmannia latifolia C. Presl & 0 & 0 & 0 & 1 & 0 & 0 \\
\hline Weinmannia lechleriana Engl. & 0 & 0 & 0 & 0 & 1 & 0 \\
\hline Weinmannia loxensis Harling & 0 & 1 & 0 & 0 & 1 & 0 \\
\hline Weinmannia multijuga Killip \& A.C. Sm. & 1 & 1 & 0 & 1 & 1 & 0 \\
\hline Weinmannia pentaphylla Ruiz \& Pav. & 0 & 0 & 0 & 0 & 1 & 1 \\
\hline Weinmannia reticulata Ruiz \& Pav. & 0 & 1 & 1 & 1 & 0 & 0 \\
\hline Weinmannia sp.1 & 0 & 0 & 1 & 0 & 0 & 0 \\
\hline Weinmannia sp.2 & 0 & 0 & 0 & 0 & 0 & 1 \\
\hline Weinmannia sp.3 & 0 & 1 & 0 & 1 & 0 & 0 \\
\hline Weinmannia sp.4 & 0 & 0 & 0 & 1 & 0 & 0 \\
\hline Weinmannia sp.5 & 0 & 1 & 0 & 1 & 1 & 0 \\
\hline Cyatheaceae & $\mathbf{0}$ & 1 & 1 & 1 & $\mathbf{0}$ & 1 \\
\hline Cyathea nephele Lehnert & 0 & 1 & 1 & 0 & 0 & 1 \\
\hline Cyathea sp.1 & 0 & 0 & 0 & 1 & 0 & 0 \\
\hline Cyathea sp.2 & 0 & 1 & 0 & 0 & 0 & 0 \\
\hline
\end{tabular}



esclerófilos de la Selva Central del Perú

\begin{tabular}{|c|c|c|c|c|c|c|}
\hline Familia/Especie & S1 & $\mathbf{S 2}$ & S3 & S4 & S5 & S6 \\
\hline Ericaceae & 1 & 1 & 1 & 1 & 1 & 1 \\
\hline Bejaria aestuans Mutis ex L. & 1 & 0 & 1 & 1 & 1 & 0 \\
\hline Cavendishia bracteata (Ruiz \& Pav. J. St. - Hill.) Hoerold & 0 & $\mathbf{1}$ & 0 & 1 & 1 & 0 \\
\hline Demosthenesia cf. buxifolia (Fielding \& Gardn.) A. C. Sm. & 0 & 0 & 0 & 0 & 0 & 1 \\
\hline Demosthenesia mandoni (Briton) A.C. Sm. & 1 & 0 & 0 & 0 & 0 & 0 \\
\hline Demosthenesia sp.2 & 0 & $\mathbf{1}$ & 0 & 0 & 1 & 0 \\
\hline Disterigma alaternoides (Kunth) Nied. & 0 & 0 & 0 & 0 & 1 & 1 \\
\hline Disterigma humboldtii (Klotzsch) Nied. & 0 & 0 & 1 & 0 & 0 & 0 \\
\hline Disterigma ovatum (Rusby) S. F. Blake & 1 & 0 & 0 & 0 & 0 & 0 \\
\hline Gaultheria aff. glomerata (Cav.) Sleumer & 0 & 0 & 1 & 0 & 0 & 0 \\
\hline Gaultheria bracteata (Cav.) G. Don & 0 & 1 & 1 & 1 & 0 & 0 \\
\hline Gaultheria vaccinioides Griseb. & 0 & 0 & 1 & 0 & 1 & 1 \\
\hline Pellegrinia cf. hirsuta (Ruiz \& Pav. Ex G. Don) Sleumer & 0 & 0 & 0 & 0 & 0 & 1 \\
\hline Pellegrinia sp.1 & 0 & $\mathbf{1}$ & 1 & 1 & 0 & 0 \\
\hline Pellegrinia sp.2 & 0 & 0 & 0 & 0 & 0 & 1 \\
\hline Thibaudia aff. crenulata Remy & 1 & 0 & 1 & 1 & 1 & 1 \\
\hline Thibaudia sp.1 & 0 & $\mathbf{1}$ & 0 & 0 & 0 & 0 \\
\hline Vaccinium dependens (G. Don) Sleumer & 1 & $\mathbf{1}$ & 1 & $\mathbf{1}$ & 1 & 1 \\
\hline Vaccinium floribundum Kunth & 1 & 1 & 1 & 0 & 1 & 0 \\
\hline Gentianaceae & 1 & 1 & 1 & $\mathbf{0}$ & 1 & 1 \\
\hline Macrocarpaea aff. ericii J. R. Grant & 0 & 0 & 0 & 0 & 1 & 0 \\
\hline Macrocarpaea pajonalis J. R. Grant & 1 & 1 & 1 & 0 & 0 & 0 \\
\hline Symbolanthus calygonus (Ruiz \& Pav.) Griseb. ex Gilg & 1 & $\mathbf{1}$ & 1 & 0 & 0 & 1 \\
\hline Hypericaceae & $\mathbf{0}$ & $\mathbf{0}$ & $\mathbf{0}$ & $\mathbf{0}$ & 1 & $\mathbf{0}$ \\
\hline Hypericum struthiolifolium Juss. & 0 & 0 & 0 & 0 & 1 & 0 \\
\hline Lauraceae & 1 & 1 & 1 & $\mathbf{0}$ & 1 & $\mathbf{0}$ \\
\hline Ocotea $\mathrm{sp} .1$ & 1 & 0 & 1 & 0 & 0 & 0 \\
\hline Ocotea $\mathrm{sp} .2$ & 0 & $\mathbf{1}$ & 0 & 0 & 1 & 0 \\
\hline Ocotea sp. 3 & 1 & 0 & 0 & 0 & 0 & 0 \\
\hline Persea pajonalis van der Werff. & 0 & 0 & 1 & 0 & 0 & 0 \\
\hline Loranthaceae & 1 & 1 & 1 & 1 & 1 & 1 \\
\hline Gaiadendron punctatum (Ruiz \& Pav.) G. Don & 1 & 1 & 1 & 1 & 1 & 1 \\
\hline Melastomataceae & 1 & $\mathbf{1}$ & 1 & 1 & 1 & 1 \\
\hline Axinaea scutigera Triana & 1 & $\mathbf{1}$ & 0 & 0 & 1 & 1 \\
\hline
\end{tabular}




\begin{tabular}{|c|c|c|c|c|c|c|}
\hline Familia/Especie & S1 & S2 & S3 & S4 & S5 & S6 \\
\hline Melastomataceae & 1 & 1 & 1 & 1 & 1 & 1 \\
\hline Brachyotum markgrafii Wurdack & 1 & 0 & 1 & 0 & 0 & 0 \\
\hline Brachyotum quinquinerve (Ruiz \& Pav.) Triana & 0 & 0 & 1 & 0 & 0 & 0 \\
\hline Miconia aff. bullata (Turcz.) Triana & 0 & 1 & 0 & 0 & 0 & 1 \\
\hline Miconia bracteolata (Bonpl.)DC. & 1 & 1 & 1 & 0 & 0 & 1 \\
\hline Miconia bullata (Turcz.)Triana & 0 & 0 & 0 & 0 & 0 & 1 \\
\hline Miconia carpishana Wurdack & 0 & 1 & 0 & 1 & 0 & 0 \\
\hline Miconia coelestis Pav. ex Naud. & 0 & 0 & 0 & 0 & 0 & 1 \\
\hline Miconia dumetosa Cogn. & 1 & 1 & 1 & 0 & 0 & 0 \\
\hline Miconia griffisii J. F. Macbr. & 0 & 1 & 0 & 0 & 0 & 1 \\
\hline Miconia latifolia (D. Don) Naudin & 0 & 1 & 0 & 0 & 0 & 0 \\
\hline Miconia micropetala Cogn. & 0 & 0 & 1 & 1 & 1 & 0 \\
\hline Miconia nigricans Cogn. & 1 & 0 & 1 & 0 & 1 & 0 \\
\hline Miconia opacifolia J. F. Macbr. & 0 & 0 & 0 & 1 & 1 & 0 \\
\hline Miconia radula Cogn. & 1 & 0 & 0 & 0 & 0 & 0 \\
\hline Miconia rugifolia Triana & 0 & 1 & 0 & 0 & 0 & 1 \\
\hline Miconia saltuensis Wurdack & 1 & 0 & 1 & 0 & 0 & 0 \\
\hline Miconia sanguinea (D. Don)Triana & 0 & 0 & 0 & 0 & 1 & 0 \\
\hline Miconia sp.1 & 0 & 0 & 0 & 0 & 1 & 0 \\
\hline Miconia theizans var. longifolia Cogn. & 1 & 0 & 0 & 0 & 0 & 0 \\
\hline Microlicia sphagnicola Gleason & 0 & 0 & 0 & 1 & 0 & 0 \\
\hline Myricaceae & $\mathbf{0}$ & $\mathbf{0}$ & $\mathbf{0}$ & 1 & $\mathbf{0}$ & $\mathbf{0}$ \\
\hline Morella pubescens (Humb. \& Bonpl. ex Willd.) Wilbur & 0 & 0 & 0 & 1 & 0 & 0 \\
\hline Myrtaceae & 1 & 1 & 1 & $\mathbf{0}$ & 1 & 1 \\
\hline Calypthranthes sp.1 & 0 & 0 & 1 & 0 & 0 & 0 \\
\hline Myrteola phylicoides var. glabrata (O. Berg) Landrum & 1 & 1 & 1 & 0 & 1 & 1 \\
\hline Pentaphylacaceae & 1 & 1 & 1 & 1 & 1 & 1 \\
\hline Freziera sp.1 & 0 & 0 & 0 & 1 & 1 & 0 \\
\hline Freziera sp. 2 & 1 & 1 & 0 & 0 & 0 & 1 \\
\hline Ternstroemia meridionalis Mutis ex. L.f. & 1 & 1 & 1 & 0 & 1 & 1 \\
\hline Ternstroemia sp.1 & 1 & 0 & 1 & 0 & 0 & 0 \\
\hline Podocarpaceae & 1 & 1 & $\mathbf{0}$ & $\mathbf{0}$ & $\mathbf{0}$ & 1 \\
\hline Podocarpus oleifolius D. Don ex Lamb. & 1 & 1 & 0 & 0 & 0 & 1 \\
\hline Polygalaceae & $\mathbf{0}$ & 1 & 1 & $\mathbf{0}$ & 1 & 1 \\
\hline
\end{tabular}




\begin{tabular}{|c|c|c|c|c|c|c|}
\hline Familia/Especie & S1 & $\mathbf{S 2}$ & S3 & S4 & S5 & S6 \\
\hline Polygalaceae & $\mathbf{0}$ & 1 & 1 & $\mathbf{0}$ & 1 & 1 \\
\hline Monina sp.1 & 0 & 0 & 1 & 0 & 0 & 0 \\
\hline Monina sp. 2 & 0 & 1 & 0 & 0 & 0 & 0 \\
\hline Monina sp.3 & 0 & 1 & 0 & 0 & 1 & 1 \\
\hline Primulaceae & 1 & 1 & 1 & 1 & 1 & 1 \\
\hline Cybianthus magnus subsp. asymmmetricus (Mez) Pipoly & 0 & 0 & 0 & 0 & 1 & 0 \\
\hline Cybianthus marginatus (Benth.) Pipoly & 1 & 1 & 0 & 0 & 0 & 0 \\
\hline Cybianthus sp.1 & 1 & 0 & 0 & 0 & 0 & 0 \\
\hline Myrsine aff. latifolia (Ruiz \& Pav.) Spreng. & 0 & 1 & 0 & 1 & 1 & 0 \\
\hline Myrsine andina (Mez) Pipoly & 0 & 1 & 1 & 0 & 0 & 1 \\
\hline Myrsine congesta (Schwacke ex Mez) Pipoly & 1 & 1 & 1 & 1 & 0 & 0 \\
\hline Myrsine coriacea (Sw.) R. Br. Ex Roem \& Schult. & 0 & 1 & 1 & 1 & 1 & 1 \\
\hline Rhamnaceae & $\mathbf{0}$ & $\mathbf{0}$ & $\mathbf{0}$ & $\mathbf{0}$ & 1 & $\mathbf{0}$ \\
\hline Rhamnus sp.1 & 0 & 0 & 0 & 0 & 1 & 0 \\
\hline Rosaceae & 1 & 1 & $\mathbf{0}$ & 1 & $\mathbf{0}$ & 1 \\
\hline Prunus ovalis Ruiz ex Koehne & 1 & 1 & 0 & 0 & 0 & 0 \\
\hline Prunus sp.1 & 0 & 0 & 0 & 0 & 0 & 1 \\
\hline Prunus sp.2 & 0 & 0 & 0 & 1 & 0 & 0 \\
\hline Rubiaceae & 1 & 1 & 1 & $\mathbf{0}$ & 1 & 1 \\
\hline Cinchona krauseana L. Andersson & 0 & 0 & 1 & 0 & 0 & 0 \\
\hline Palicourea aphthosa Standley & 0 & 1 & 0 & 0 & 1 & 0 \\
\hline Psychothria sp.1 & 0 & 1 & 0 & 0 & 0 & 1 \\
\hline Psychothria sp.2 & 0 & 0 & 1 & 0 & 0 & 0 \\
\hline Psychothria virgata Ruiz \& Pav. & 1 & 0 & 0 & 0 & 0 & 0 \\
\hline Smilacaceae & $\mathbf{0}$ & 1 & 1 & $\mathbf{0}$ & $\mathbf{0}$ & $\mathbf{0}$ \\
\hline Smilax domingensis Willd. & 0 & 1 & 1 & 0 & 0 & 0 \\
\hline Styracaceae & $\mathbf{0}$ & $\mathbf{0}$ & $\mathbf{0}$ & $\mathbf{0}$ & $\mathbf{0}$ & $\mathbf{1}$ \\
\hline Styrax foveolaria Perkins & 0 & 0 & 0 & 0 & 0 & 1 \\
\hline Symplocaceae & $\mathbf{0}$ & 1 & 1 & $\mathbf{0}$ & 1 & 1 \\
\hline Symplocos alpina Brand & 0 & 0 & 0 & 0 & 0 & 1 \\
\hline Symplocos cf. apiciflora B. Stahl & 0 & 0 & 0 & 0 & 0 & 1 \\
\hline Symplocos coriacea A. DC. & 0 & 1 & 1 & 0 & 0 & 1 \\
\hline Symplocos fimbriata B. Stahl & 0 & 1 & 0 & 0 & 0 & 0 \\
\hline Symplocos nana Brand & 0 & 0 & 0 & 0 & 0 & 1 \\
\hline Symplocos sp.1 & 0 & 0 & 0 & 0 & 1 & 0 \\
\hline
\end{tabular}

